\title{
BMJ Open Outcomes comparison of different surgical strategies for the management of severe aortic valve stenosis: study protocol of a prospective multicentre European registry (E-AVR registry)
}

To cite: Onorati F, Gherli $\mathrm{R}$, Mariscalco G, et al. Outcomes comparison of different surgical strategies for the management of severe aortic valve stenosis: study protocol of a prospective multicentre European registry (E-AVR registry). BMJ Open 2018:8:e018036. doi:10.1136/ bmjopen-2017-018036

- Prepublication history and additional material for this for this paper are available online. To view these files, please visit the journal online (http://dx.doi org/10.1136/bmjopen-2017018036).

Received 1 June 2017 Revised 17 December 2017 Accepted 18 December 2017

Check for updates

For numbered affiliations see end of article.

Correspondence to Dr Francesco Onorati; francesco.onorati@aovr.veneto. it

\section{ABSTRACT}

Introduction Traditional and transcatheter surgical treatments of severe aortic valve stenosis (SAVS) are increasing in parallel with the improved life expectancy. Recent randomised controlled trials (RCTs) reported comparable or non-inferior mortality with transcatheter treatments compared with traditional surgery. However, RCTs have the limitation of being a mirror of the predefined inclusion/exclusion criteria, without reflecting the 'real clinical world'. Technological improvements have recently allowed the development of minimally invasive surgical accesses and the use of sutureless valves, but their impact on the clinical scenario is difficult to assess because of the monocentric design of published studies and limited sample size. A prospective multicentre registry including all patients referred for a surgical treatment of SAVS (traditional, through full sternotomy; minimally invasive; or transcatheter; with both 'sutured' and 'sutureless' valves) will provide a 'real-world' picture of available results of current surgical options and will help to clarify the 'grey zones' of current guidelines.

Methods and analysis European Aortic Valve Registry is a prospective observational open registry designed to collect all data from patients admitted for SAVS, with or without coronary artery disease, in 16 cardiac surgery centres located in six countries (France, Germany, Italy, Spain, Switzerland and UK). Patients will be enrolled over a 2-year period and followed up for a minimum of 5 years to a maximum of 10 years after enrolment. Outcome definitions are concordant with Valve Academic Research Consortium-2 criteria and established guidelines. Primary outcome is 5-year all-cause mortality. Secondary outcomes aim at establishing 'early' 30-day all-cause and cardiovascular mortality, as well as major morbidity, and 'late' cardiovascular mortality, major morbidity, structural and non-structural valve complications, quality of life and echocardiographic results.
Strengths and limitations of this study

- The protocol addresses the important question of which surgical treatment offers the most benefits in the management of patients with severe aortic valve stenosis, with or without concomitant coronary artery disease. The expected large sample size will guide subanalyses aimed at identify specific patient characteristics and different risk profiles, which are better served with alternative surgical techniques.

- The minimum 5-year and maximum 10-year followup will provide answers about the mid-to-long term safety and efficacy of recent surgical innovations (ie, sutureless valves, minimally invasive approaches, surgical transcatheter aortic valve replacement), whose follow-up data are still lacking in current literature.

- The present multicentre registry has clearly established aim, inclusion and exclusion criteria, short-term and follow-up primary and secondary endpoints, as well as state-of-the-art methods for data collection and endpoints definition.

- Limitations include variations in postoperative and follow-up management, which are based on local Institutional policies, and lack of blinding between the central statistical core lab performing the analyses and the employed surgical techniques.

- The absence of any external sponsor certainly limits research resource allocation but also guarantees the certainty for the absence of any bias or conflict of interest related to the investigated topics.

Ethics and dissemination The study protocol is approved by local ethics committees. Any formal presentation or publication of data will be considered as a joint publication by the participating physician(s) and will follow the recommendations of the International Committee of Medical Journal Editors for authorship.

Trial registration number NCT03143361; Pre-results. 


\section{INTRODUCTION}

The prevalence of severe aortic valve stenosis (SAVS) correlates with age, and its treatment is expected to increase parallel to the improved life expectancy reported in western countries. ${ }^{12}$ Traditional surgical aortic valve replacement (SAVR) has been the gold standard of treatment for decades, with well-documented benefits in terms of symptom improvement and survival. ${ }^{34}$ Recent technological advances allowed interventional and surgical transcatheter aortic valve replacements (TAVR), which proved to be effective alternatives to traditional SAVR, in both high-risk and intermediate-risk patients. ${ }^{5-12}$ Therefore, SAVR and TAVR represent nowadays the standard surgical armamentarium for aortic valve replacement.

Alternative surgical approaches, based on the concept of TAVR, ${ }^{1314}$ have in fact been developed. There are on the market two types of 'sutureless' valves (ie, Sorin Perceval and Edwards Intuity) at the moment-aimed at reducing some surgical drawbacks such as cross-clamp time and myocardial ischaemia-reperfusion injury ${ }^{13}{ }^{15-20}$-and it is possible that new 'sutureless' valves will enter the market in the next future. Moreover, different minithoracotomy and ministernotomy approaches to SAVR have been widely adopted by the surgical community-with both 'sutured' and 'sutureless' valves-in order to reduce surgical trauma, systemic inflammatory response and major organ morbidity. ${ }^{1315} 16$ Various different combinations of minimally invasive accesses and the use of last-generation valves have been reported to date. ${ }^{1417} 18$ However, despite early enthusiasm about preliminary results with these technological improvements, none of these techniques has yet replaced traditional SAVR in standard surgical practice, mainly because reporting of results of these alternative techniques tends to be biased by singlecentre design, limited sample size and the strict inclusion/exclusion criteria of the published studies. ${ }^{13} 14$

Another 'hot topic' in this debate relates to valve durability, given that the long-term durability of both TAVR and sutureless valves is as yet unknown. Indeed, standard 'sutured' surgical valves demonstrated excellent durability, both in the very long term and in very young adults below the 65-year cut-off age, ${ }^{21-23}$ which is still the threshold for biological valves recommended by European Society of Cardiology/European Association for CardioThoracic Surgery guidelines. ${ }^{24}$ This issue is of particular interest, given that the use of TAVR has increased in younger intermediate-risk patients, despite recent caveats relating to early degeneration of TAVR. ${ }^{25} 26$

Similarly, improved life expectancy has led to a growing number of patients with degenerated dysfunctioning aortic bioprostheses requiring surgical treatment. Again, surgical aortic prosthetic replacement has been traditionally considered the only treatment strategy for these patients, given the excellent results, recently confirmed by several studies. ${ }^{27} 28$ Again, transcatheter treatments ('valve-in-valve') have recently demonstrated comparable or sometimes superior results in redo scenarios. ${ }^{29}$ Therefore, data on the efficacy, safety and durability of these technological improvements are essential for providing 'strong' statements in future guidelines and for evaluating the extension of these techniques to low-risk and young patients in the future.

Finally, it is well known that critical coronary artery disease (CAD) often coexists with SAVS. Although the standard treatment option for these patients has traditionally been SAVR plus coronary artery bypass grafting (CABG), the introduction of TAVR and minimally invasive surgical alternatives has considerably changed the available options, paving the way to combined less invasive SAVR+CABGand/or TAVR \pm Percutaneous coronary intervention (PCI) (either before, during or after the surgical procedure) ${ }^{30-34}$ Again, there is a great deal of confusion on the topic, and there are unanswered questions on the efficacy and safety of these options, as well as on what to expect from late follow-up when compared with the standard practice of SAVR+CABG.

Robust early and follow-up data on the safety and efficacy of surgical TAVR, last-generation sutureless surgical valves and minimally invasive approaches compared with standard SAVR, with or without a contemporary (surgical or interventional) treatment of concurrent CAD, are still lacking for a real-world large population of patients at variable surgical risk. Such data are urgently required for the correct allocation of therapy in daily surgical practice. Furthermore, data on quality of life (QoL) and functional echocardiographic results with different surgical alternatives might similarly help physicians in decision making in local 'Heart Teams'. Data from a multicentre, real-world, open registry enrolling all patients with SAVS \pm CAD consecutively referred to several centres at different European latitudes should help to answer some of these open questions. Here, we describe the rationale and the study protocol of the European Aortic Valve Registry (E-AVR), a multicentre prospective observational open registry on aortic valve surgical practice.

\section{METHODS AND ANALYSIS}

\section{Rationale of the study and aim}

Improvements in surgical treatment of cardiac diseases can be obtained with the implementation of current techniques and the development of new methods, based on information from large clinical datasets. ${ }^{35}$ The main strength of a prospective clinical open registry is the high external validity, given that data are collected in the settings of standard clinical practice. Moreover, large sample size enables a better estimation of event rates and allows the investigation of hard endpoints and outcomes by means of a wide population of patients from different institutions and with extremely limited exclusion criteria.

Importantly, clinical registries may provide data on long-term outcomes occurring after the study period of a trial. ${ }^{35}$ They are more practical than randomised controlled trials, require fewer resources and have less stringent inclusion and exclusion criteria for patient enrolment. Finally, clinical findings from registries have 


\section{European Cardiac Surgery Centres}

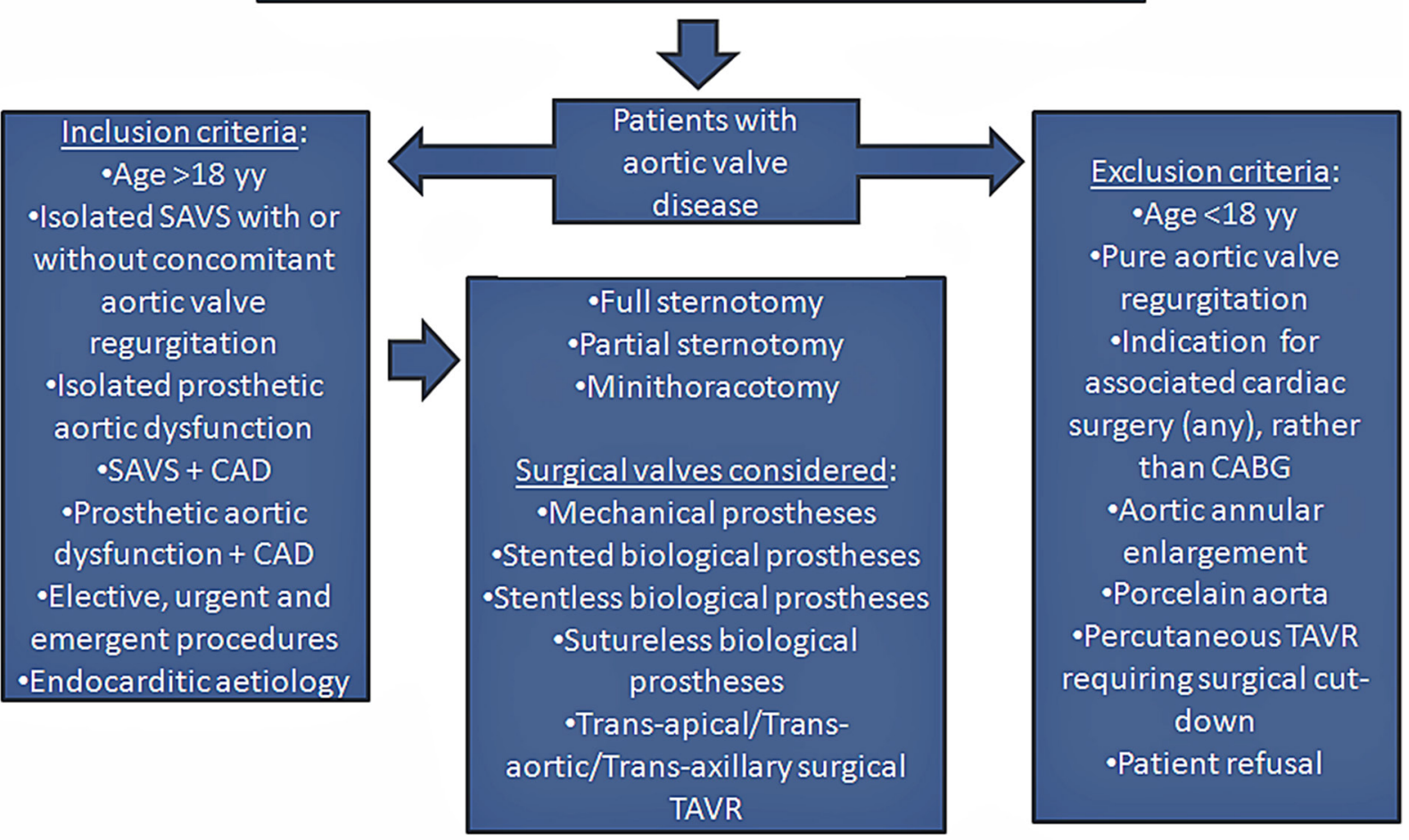

Figure 1 Flow chart of enrolment criteria and surgical techniques considered in the registry. CABG, coronary artery bypass grafting; CAD, coronary artery disease; SAVS, severe aortic valve stenosis; TAVR, transcatheter aortic valve replacement.

even more significance when patient populations derive from different geographic areas, with heterogeneous referral pathways, baseline clinical characteristics and perioperative treatment strategies. All these features substantiate the concept of 'a real world practice' underlying any 'registry-study'.

Therefore, the rationale of this European multicentre observational open registry is to prospectively collect data on baseline characteristics, treatment options, perioperative management and postoperative outcome of all patients consecutively undergoing surgical treatment of SAVS (regardless of gradients, aortic valve area (AVA) or Indexed Aortic Valve area (AVAi) $\pm \mathrm{CAD}$ or aortic prosthetic dysfunction $\pm \mathrm{CAD}$ at 16 European university or non-university tertiary hospitals located in six European countries (France, Germany, Italy, Spain, Switzerland and UK). The complete list of E-AVR Collaborators is reported in the online supplementary appendix.

The primary aim of the study is a 5-year comparison between SAVR and surgical TAVR; we hypothesise to report a $10 \%$ inferior 5-year all-cause mortality event rate in SAVR, that is, we expect that SAVR survival will exceed by $10 \%$ (absolute value) that of surgical TAVR. For the purpose of this study, patients will be consecutively enrolled for a 2-year period and will be followed up for a minimum of 5 years after the index surgical treatment. Maximum follow-up length will be 10 years after surgery.

The following surgical options will be considered:

1. SAVR with mechanical valves
2. SAVR with biological valves (either sutured or sutureless, stented or stentless)

3. surgical TAVR (either transapical, transaxillary or transaortic).

Similarly, the following surgical approaches will be considered:

1. full sternotomy

2. minithoracotomy (either left sided for TAVR or right sided for SAVR)

3. partial sternotomy.

Patient allocation to a specific surgical procedure will be based on the local Heart Team decision at each Institution, according to standard clinical practice and current guidelines. $^{2}$

A flow chart of the enrolment criteria and of the surgical techniques considered in the registry is provided in figure 1.

\section{Criteria for registry enrolment}

The following inclusion and exclusion criteria will be considered.

Inclusion criteria

- Age $>18$ years old

- isolated SAVS with or without concomitant aortic valve regurgitation

- isolated prosthetic aortic dysfunction

- SAVS+CAD

- prosthetic aortic dysfunction+CAD 


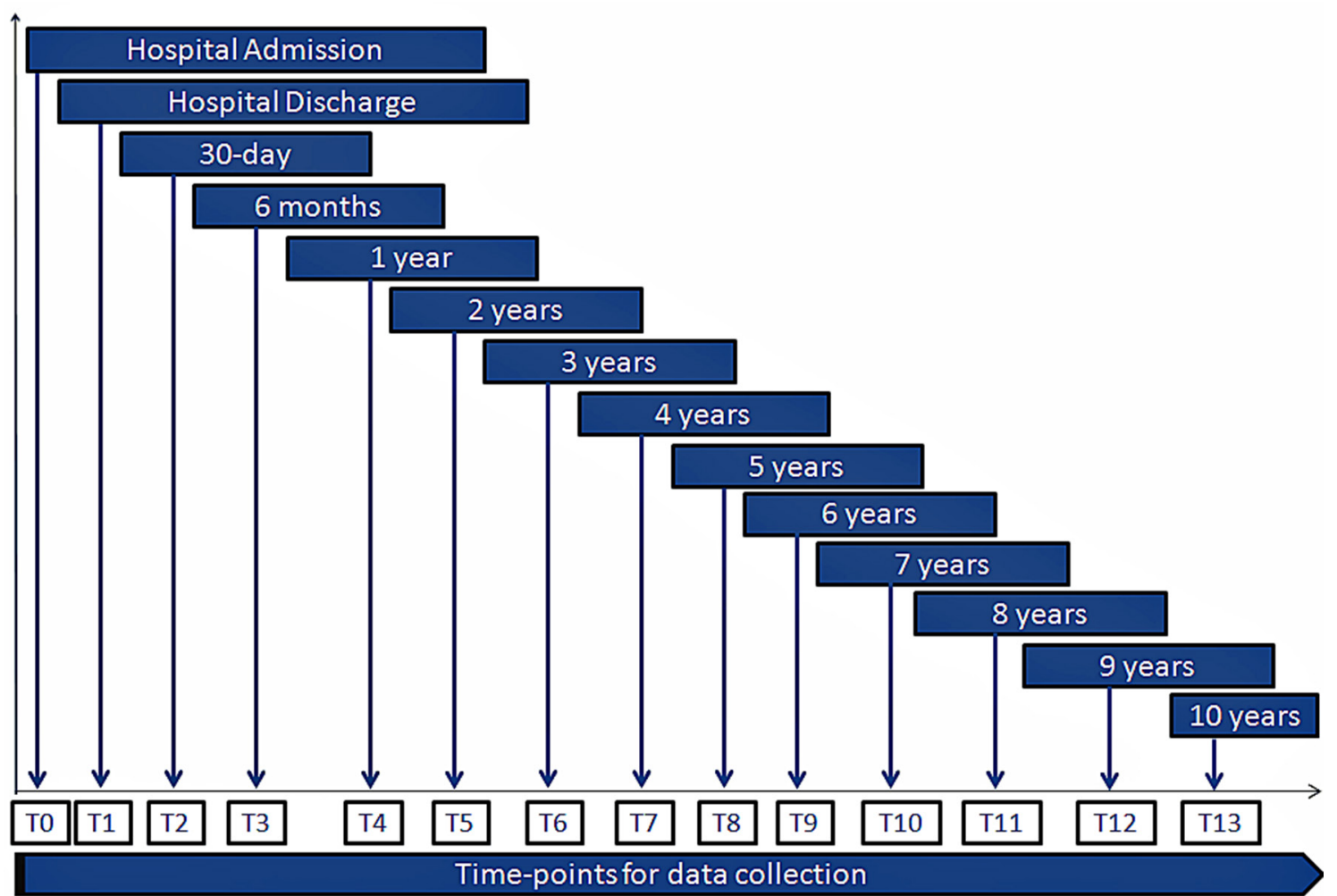

Figure 2 Flow chart of time-points for data collection.

- elective, urgent and emergent procedures

- endocarditic aetiology.

Exclusion criteria

- Patients undergoing concomitant mitral valve surgery, or tricuspid valve surgery, or aortic surgery (ie, composite aortic valve and ascending aorta replacement with o without circulatory arrest), or atrial fibrillation surgery, or any other associated cardiac surgical procedure (with the exception of CABG)

- concomitant aortic root procedure (ie, Bentall operation, David operation, homografts and autografts)

- SAVR with techniques of aortic annular enlargement

- porcelain aorta

- pure aortic valve regurgitation

- percutaneous TAVR (regardless of technique, ie, either by a completely percutaneous approach or by surgical cut-down)

- patient refusal.

Patients will be recruited in a consecutive series from each institution, and their data will be collected in a dedicated online datasheet. The recruitment period will be 24 months, from 1 November 2017 to 30 October 2019. Every patient will be followed up at 30 days, 6 months, 1 year and yearly thereafter, up to a minimum of 5 years after the index surgical procedure (figure 2). Afterwards, yearly follow-up will be closed at the completion of the 10th year from surgery for each patient.

On the basis of historical cohort data of local institutions, we expect to enrol a minimum of 4000 patients at the end of the first year and a minimum of 8000 patients at the end of the second year of enrolment.

\section{Informed consent}

Written informed consent will be obtained from the patient or patient's authorised representative prior to enrolment in the registry. In case of emergent surgery, informed consent will be collected from the patient's family (or legal representative) before surgery, as well as from the patient after surgery (if unable to give it before intervention). This consent will be waived in case of death or severe neurological damage precluding adequate postoperative patient informed consent. The study will be conducted in accordance with the provisions of the Declaration of Helsinki. The study is registered in Clinicaltrials.gov. (no. NCT03143361).

\section{Data management and monitoring}

Data will be collected into a dedicated datasheet with predefined variables. Each patient enrolled in the registry will be anonymised by the generation of a code consisting of the initials of the enrolling country (two letters), enrolling centre (two letters) and then consecutive number (considered at thousands) (eg, Mr XY, third patient enrolled in London=UKLO0003). It is the responsibility of the E-AVR Steering Committee local member to generate the sequence to maintain anonymised the entire set of data. It is also the responsibility of the E-AVR Steering Committee local member to protect confidentiality about patient identity before, during and after the trial. Accordingly, the external Central Statistical Core 
Lab (as well as all the other E-AVR investigators) will be blinded towards patient identity.

All data will be retained in a secure location at each study site during the conduct of the study and for the 5 years after the end of the study, when all patient identifiable paper records will be destroyed by confidential means.

Baseline characteristics, operative details and outcome data pertaining hospitalisation will be prospectively collected from hospital registries. Variables and events occurring after the index hospital discharge will be collected from outpatient clinics at the individual Institutions, and linking with regional Social Security Death and Events Master files where available. In case of absent/ missing data, variables and events will be collected by direct phone contact with general practitioners and only if persistently missed by phone contact with patients and families.

Events and outcome variables will be adjudicated centrally by a Central Core Lab (Unit for Clinical Research and Biostatistics, Verona University Hospital, Verona, Italy). In the event of controversy on outcome adjudication, this will be discussed and adjudicated after a final consult between the Central Core Lab and the E-AVR Steering Committee.

Storage, analysis and auditing of data will be also accomplished by the independent Central Core Lab. Auditing of the dataset will be performed every 6 months by checking the data of a minimum of $40 \%$ of the patients. Data without any patient identification code will be submitted to the principal investigator (PI) and E-AVR Steering Committee for further data checking and merging. Incomplete or contradictory data with patient identification code will be sent from Central Core Lab to the E-AVR Steering Committee local member for further data checking, review, correction and merging. The entire set of statistical analyses will be available to all E-AVR researchers for the interpretation of data.

\section{Statistical methods}

The Central Core Lab (Unit for Clinical Research and Biostatistics, Verona University Hospital, Verona, Italy) will perform all the statistical analyses derived from this registry.

It is intended to enrol 8000 patient, of which $60 \%-70 \%$ will be SAVR and the remainder TAVR (historical data based on institutional practices). Considering the estimated event rate of $25 \%$ in the TAVR patients at 5 years, ${ }^{36}$ a 2-year accrual time, an anticipated loss-to-follow-up rate of $1.5 \%$ (historical data) and the target power of $80 \%$ at a 0.05 one-sided log-rank test significance level to detect the hypothesised $10 \%$ inferior (absolute improvement) 5-year all-cause mortality rate in favour of SAVR, the calculations showed the overall sample size of the registry to meet the targeted power for all expected scenarios of estimated proportions of SAVR and TAVR patients.

Similar calculation shows that, considering the estimated 30-day all-cause mortality event rate of $5.0 \%$ after
SAVR and of $5.5 \%$ after TAVR ( $0.5 \%$ absolute difference $)$ in intermediate-risk patients, ${ }^{37}$ the overall sample size of the registry also meets the targeted $80 \%$ power to detect a difference as small as $0.5 \%$ in 30-day all-cause mortality event rate in favour of SAVR. Other secondary endpoints will serve as exploratory analyses, possibly useful for sample size estimation of future clinical trials.

Statistical calculations were accomplished with PASS 14.0 Power Analysis and Sample Size Software (2015) statistical package (NCSS, LLC; Kaysville, Utah, USA; ncss.com/software/pass).

Continuous variables will be reported as mean and SD or median and IQR, as appropriate. Dichotomous and nominal variables will be reported as counts and percentages. Univariate analysis will be performed using the Mann-Whitney U test and Student's t-test for continuous variables (pending the not-normal or normal distribution, respectively), the Kruskal-Wallis test (independent multilevel ordinal variables), Wilcoxon test (for paired variables), Fisher's exact test and $\chi^{2}$ test (for dichotomous/nominal variables) and Kaplan-Meier test (for time-dependent dichotomous variables). Log-rank test will be used to compare the 5-year all-cause mortality rate between SAVR and surgical TAVR. Multivariable analyses will be performed using logistic regression method (for categorical dependent variable), classification tree analysis (for target variables with a discrete set of value), linear regression (for continuous dependent variable) and ordinal regression methods (for ordinal dependent variable). Cox proportional hazards method will test the effects of covariates on time-dependent dichotomous variables; the model's proportional hazard assumption will be checked using the Schoenfeld residuals test. Significant differences between study groups will be adjusted by using propensity score as covariate or by one-to-one propensity score matching. Matching will be performed using a calliper width of 0.2 of the SD of logit of the propensity score. Multiple propensity score adjusted analysis will be performed in case of multiple study groups. A Bayesian hierarchical approach will be used in the case of significant variability between centres.

Missing values will be replaced and estimated using multiple imputations. Furthermore, sensitivity analysis will be executed using complete-case analysis. Interim analyses are planned at different time-points (see Ethics and dissemination). Critical $\mathrm{P}$ values of accomplished interim analyses will be corrected according to the Armitage-McPherson adjustment. ${ }^{38}$

\section{Early and late endpoints}

Outcome endpoints will be defined according to current guidelines, that is, Valve Academic Research Consortium-2 (VARC-2) definitions ${ }^{39}$ and guidelines for reporting mortality and morbidity after cardiac valve interventions. ${ }^{40}$

In more detail, the following outcome variables will be collected: 
Primary outcome of the E-AVR registry

Five-year all-cause mortality.

\section{Secondary outcomes of the E-AVR registry}

These will be dichotomised into 'early' at 30 days (ie, during hospitalisation, at home if discharged or during 'rehab-hospitalisation' at any time-point if never discharged home) and 'late' (after the patient is discharged home):

1. Early secondary outcomes: all-cause mortality, cardiovascular mortality, stroke, acute myocardial infarction $(\mathrm{AMI}){ }^{41}$ postoperative need for prolonged use of inotropes ( $>72$ hours), postoperative need for intra-aortic balloon pump (IABP) or extracorporeal mechanical oxygenation (ECMO), surgical site infection, blood losses and use of blood products (during hospitalisation for the index surgical procedure), nadir haematocrit, nadir haemoglobin, resternotomy for bleeding, atrial fibrillation (first event and number of events), cardiac conduction disturbances, need for new permanent pacemaker implantation, acute kidney injury (following acute kidney injury (AKIN) classification), pericardial effusion requiring treatment, length of stay in the intensive care unit, length of inhospital stay (for the index procedure), device success, early safety, clinical efficacy, time-related valve safety, echocardiographic data of prosthesis performance and early repeat surgery for failure of the index procedure (any 'redo' before discharge home or to rehabilitation clinic).

2. Late secondary outcomes (collected starting from discharge to the end of the 10th year after the index procedure): cardiovascular mortality, all-cause mortality (from 1 year to 4 years after surgery, then from 6 years to 10 years), stroke, AMI, reintervention on the aortic prosthesis, repeat revascularisation (either with percutaneous coronary intervention or $\mathrm{CABG}$ ), prosthetic thrombosis, embolism, bleeding events, structural valve deterioration, paravalvular leakage, prosthetic endocarditis, need for permanent pacemaker, need for implantable cardioverter-defibrillator, Major Cardiovascular and Cerebrovascular Events (MACCE) (defined as a composite endpoint including any of the following adverse events: death from cardiovascular cause, stroke, myocardial infarction and repeated revascularisation), time-related valve safety, QoL defined according to Short Form-8 (SF-8) questionnaire; QoL will be assessed during follow-up visits at outpatient clinics or, if other methods are not possible, by telephone interview); echocardiographic data of prosthesis performance.

Echocardiographic data of prosthesis performance are defined according to the VARC-2 definitions. ${ }^{38}$ All echocardiographic data will be collected from third level nationally and/or internationally certified Institutional Echo Laboratories: $5 \%$ of these echocardiographic exams will be reviewed centrally (Unit for Clinical Research and Biostatistics, Verona University Hospital, Verona, Italy) by third-level certified echocardiographers. Collection of data is under the responsibility of the steering committee local member at each participating centre. Data will be audited from the Central Core Lab on a regular basis, as reported previously.

Outcomes and their definition criteria are described in detail in the following section of this article.

\section{Data collection}

\section{Participating centre}

Each participating centre will be anonymised by identification with a capital letter. The correspondence between centres and capital letters will only be known by the PI of the study. The Central Core Lab analysing the data will be blinded towards the surgical teams.

\section{Units of measurement}

Laboratory data will be collected according to fixed units of measurement in order to avoid any problem stemming from differences in units used by the various participating centres, during data merging and analysis. The fixed units of measurement are reported in the dedicated case record form (CRF) datasheet.

\section{Laboratory parameters}

Baseline levels of haemoglobin, haematocrit, platelets, blood glucose, Glycated Hemoglobin (HbA1c), C reactive protein, International Normalized Ratio (TT-INR) and albumin will be collected.

\section{Hypertension}

Arterial blood pressure $>140 / 90 \mathrm{~mm} \mathrm{Hg}$ or antihypertensive treatment.

\section{Diabetes}

Diabetes mellitus requiring diet, oral or insulin treatment.

\section{Preoperative creatinine levels}

This parameter is obtained on the day before surgery and is expressed in $\mu \mathrm{mol} / \mathrm{L}$.

\section{Chronic kidney disease}

The severity of renal failure will be classified as shown in table 1 . It is stratified by the estimated glomerular filtration rate (eGFR) calculated using the Modification of Diet in Renal Disease Study Group modified formula. ${ }^{42}$ eGFR for calculation of the EuroSCORE II $^{43}$ will be

\begin{tabular}{ll}
\hline Table 1 & Stages of renal failure \\
\hline Stages & eGFR level $\left(\mathbf{m L} / \mathbf{m i n} / \mathbf{1 . 7 3} \mathbf{~ m}^{2}\right)$ \\
\hline 1 & 90 or above \\
\hline 2 & 89 to 60 \\
$3 \mathrm{a}$ & 59 to 44 \\
$3 \mathrm{~b}$ & 44 to 30 \\
4 & 29 to 15 \\
5 & Less than 15 or on dialysis \\
\hline
\end{tabular}


estimated using the Cockcroft-Gault formula ${ }^{44}$ according to the criteria of this risk scoring method.

Dialysis

Peritoneal or haemodialysis before surgery.

Chronic obstructive pulmonary disease

Any long-term use of bronchodilators or steroids for lung disease.

\section{Oxygen therapy}

Long-term oxygen therapy for respiratory failure.

\section{Liver disease}

Different degrees of liver failure stratified according to the Child-Pugh classification. ${ }^{45}$

\section{Active neoplasia}

Any active malignancy.

\section{Preoperative stroke}

Any preoperative focal or global neurological syndrome caused by ischaemia or haemorrhage not resolving within 24 hours.

\section{Neurological dysfunction}

Disabling outcomes in ambulation and/or normal motor functions, according to EuroSCORE II definition. ${ }^{43}$

\section{Extracardiac arteriopathy}

One or more of the following: claudication, carotid occlusion or $>50 \%$ stenosis, amputation for arterial disease, previous or planned intervention on the abdominal aorta, limb arteries or carotids.

\section{Preoperative ECG}

Sinus rhythm or atrial fibrillation, or first degree atrio-ventricular (AV) block, or right bundle block, or left bundle block or pacemaker rhythm.

Preoperative myocardial infarction

Any preoperative myocardial infarction.

\section{Previous vascular surgery}

History of surgical or endovascular procedure of the thoracic or abdominal aorta and/or the iliac-femoral arteries.
Previous cardiac surgery

One or more previous cardiac operations requiring opening of the pericardium.

Type of previous cardiac surgery

Description of previous cardiac operation.

Previous aortic valve replacement

Description of prosthesis and date of operation.

Previous percutaneous coronary intervention

Any previous percutaneous coronary intervention.

Aetiology of aortic valve disease

Native valve disease (degenerative, rheumatic and endocarditic) or prosthetic valve disease.

\section{Endocarditis}

Any diagnosis of valve endocarditis made by the Heart Team and/or antibiotic treatment for endocarditis at the time of surgery. Subclassification into acute, subacute and healed endocarditis based on current guidelines will be added. ${ }^{46}$

\section{Endocarditis aetiology}

Microbe isolated for the diagnosis of endocarditis.

New York Health Association (NYHA) functional classes

Defined according to the criteria listed in table $2 .{ }^{47}$

\section{Aortic valve stenosis}

Severity of aortic valve stenosis before surgery will be graded as moderate or severe according to 2014 American Heart Association (AHA)/American College of Cardiology (ACC) guidelines for the management of patients with valvular heart disease. ${ }^{48}$

\section{Aortic valve regurgitation}

Severity of aortic valve regurgitation before surgery will be graded in classes from 0 to 3 , and the grade of severity will be evaluated according to 2014 AHA/ACC guidelines for the management of patients with valvular heart disease. ${ }^{48}$

Associated critical CAD

Presence of stenosis of at least $70 \%$ in any major epicardial coronary artery. Number of main vessels involved will be recorded. Patients with stenosis of the left main

\section{Table 2 New York Heart Association functional classes}

\section{Class Definition}

I Cardiac disease, but no symptoms and no limitation in ordinary physical activity, for example, no shortness of breath when walking, climbing stairs and so on.

II Mild symptoms (mild shortness of breath and/or angina) and slight limitation during ordinary activity.

III Marked limitation in activity due to symptoms, even during less-than-ordinary activity, for example, walking short distances (20-100 m). Comfortable only at rest.

IV Severe limitations. Experiences symptoms even while at rest. Mostly bedbound patients. 
coronary artery will be considered as having at least two-vessel disease.

Associated left main CAD

Any LMSD $>50 \%$.

\section{Mitral valve regurgitation}

Severity of concurrent mitral valve regurgitationthough not requiring surgery-will be graded in classes according to 2014 AHA/ACC guidelines for the management of patients with valvular heart disease. ${ }^{48}$

Left ventricular (LV) function: last measured LV ejection fraction before surgery (in any case before induction of anaesthesia).

\section{Pulmonary hypertension}

Absent: <31 mm Hg; moderate: 31-55 mm Hg; severe: $>55 \mathrm{~mm} \mathrm{Hg}$, according to EuroSCORE II definition. ${ }^{43}$ Systolic pulmonary pressure will be estimated at echocardiography, at least before induction of anaesthesia.

\section{Preoperative echocardiography data}

Aortic valve area, peak transvalvular gradient, mean transvalvular gradient, aortic annulus diameter, maximum jet velocity (transthoracic echocardiography (TTE) will be recorded.

\section{Preoperative multislice CT scan data}

Annulus circumference, valvular area and calcium score (collected for all surgical TAVR, and only if available for other surgical techniques).

\section{Diseased ascending aorta}

Any sign of diffuse atherosclerosis in the ascending aorta at palpation or epiaortic ultrasound (porcelain aorta is not considered).

\section{Montgomery classification}

If available, echocardiographic Montgomery classification of aortic atheromas will be provided.

\section{Preoperative antithrombotic or antibiotic drug treatment}

Data on all antithrombotic drugs administered before surgery will be collected. The date of pause of drug treatment is the last day the patient received the drug. Data on any oral or intravenous antibiotics administered preoperatively without prophylaxis purpose, that is, for any preoperative infectious condition, will be collected.

\section{Elective surgery}

Elective procedure scheduled for stable aortic valve disease.

\section{Urgent surgery}

Procedure indicated by medical factors that require the patient to stay in hospital to have operation before discharge.
Emergency surgery

Procedure performed before the beginning of the working day after the decision to operate.

Frailty

Preoperative patient's frailty is graded according to Geriatric Status Scale, as proposed by Rockwood et al. ${ }^{49}$

\section{Critical preoperative status}

Ventricular tachycardia or ventricular fibrillation or aborted sudden death, preoperative cardiac massage, preoperative ventilation before anaesthetic room, preoperative inotropes or IABP and preoperative acute renal failure (anuria or oliguria $<10 \mathrm{~mL} /$ hour) according to EuroSCORE II definition. ${ }^{43}$

\section{EuroSCORE ॥}

This risk score is calculated using the on-line calculator available at http://www.euroscore.org/calc.html and reported as a percentage. The risk factors included in the EuroSCORE II and collected in the E-AVR registry are defined according to the EuroSCORE II criteria. ${ }^{42}$

\section{STS score}

This risk score is calculated using the online calculator available at http://riskcalc.sts.org/stswebriskcalc/\#/ and reported as a percentage. The risk factors included in the Society of Thoracic Surgeons (STS) score and collected in the E-AVR registry are defined according to the STS score criteria. ${ }^{50}$

\section{Surgical chest access}

Classified as (1) full sternotomy, (2) minithoracotomy and (3) partial sternotomy.

\section{Aortic valve replacement data}

Classified as (1) mechanical prosthesis, (2) stented biological prosthesis, (3) stentless biological prosthesis, (4) sutureless biological prosthesis, (5) transapical TAVR and (6) transaortic TAVR. The description of model and diameter of the prosthesis implanted and possible need for proctored procedure will also be collected.

\section{Other intraoperative data}

Type of cardioplegia and its temperature, duration of extracoroporeal circulation (ECC), nadir temperature of ECG and aortic cross-clamping time, need for re-aortic cross-clamping for any reason (paravalvular leak, coronary obstruction, annular rupture/ haematoma, reconstruction of CABG and so on), as well as details of TAVR implantation including sheath size, preimplantation valvuloplasty, occurrence of valve-in-valve emergency procedure, the number of valves implanted, prosthesis migration, recapturing and repositioning of the valve, postprocedural dilation and amount of contrast medium administered will be collected. 


\section{CABG details}

Details of types of conduit and target vessel will be reported (eg, LIMA-LAD, RIMA-Dx, RA-MO and SV-DIAG). The following specifications for conduits will be used: LIMA: left internal mammary artery; RIMA: right internal mammary artery; RA: radial artery; GEA: gastro-epiploic artery; SV: saphenous vein. The following target acronyms will be used: DA: anterior descending; DIAG: diagonal; RX: right coronary (trunk); PDA: posterior descending; PL: posterolateral; OM: obtuse marginal. In the event of sequential grafting, the prefix 'seq' will be used before targets (eg, LIMA-seq DIAG-DA).

\section{Other CABG details}

Number of distal anastomoses, completeness of revascularisation.

\section{0-day all-cause mortality}

Defined as the sum of cardiovascular and non-cardiovascular, the latter defined as any death in which the primary cause is clearly related to another condition not contemplated by the definition 'cardiovascular' (eg, trauma and cancer), as in VARC-2 definition, ${ }^{39}$ but occurring within 30 days or during index procedure hospitalisation if the postoperative length of stay is longer than 30 days.

\section{0-day cardiovascular mortality}

Based on VARC-2 definition ${ }^{39}$ and occurring within 30 days or during hospitalisation for the index procedure if the postoperative length of stay is longer than 30 days. This includes: (1) death due to proximate cardiac cause (eg, myocardial infarction, cardiac tamponade, worsening heart failure and low cardiac output syndrome); (2) death caused by non-coronary vascular conditions (eg, pulmonary embolisms, stroke, aortic rupture or vascular dissection); (3) all procedure-related deaths (including those related to a complication of the procedure or a treatment for a complication of the procedure); (4) all valve-related deaths including valve dysfunction (structural or non-structural) and other valve-related adverse events; and (5) sudden or unwitnessed death.

Type 5 myocardial infarction

Defined according to the recent criteria defined by Moussa et a $\tilde{p}^{1}$ (table 3).

Atrial fibrillation

Any new paroxysmal/permanent atrial fibrillation episode requiring or not requiring pharmacological or electrical cardioversion attempts. Number of recurrences will be also collected.

\section{Cardiac conduction disturbances}

Defined as a new left bundle branch block, right bundle branch block or AV block (first, second or third degree). Diagnosis will be based on official medical reports from a consultant cardiologist. In case of progressive bradiarrhythmias, the evolution of the arrhythmias will be collected.

\section{Need for permanent Pacemaker}

Collected as a dichotomic variable. Type of permanent pacing set-up (eg, Atrio-Atrial Inhibited (AAI), Ventricular-ventricular inhibited (VVI) and Dual chamber sensing/pacing (DDD)) will be collected.

\section{Postoperative neurological damage}

Classified as: (0) absent, (1) disabling stroke, (2) non-disabling stroke and (3) TIA, based on definitions of VARC-2 consensus. $^{39}$

\section{Stroke classification}

(1) Ischaemic, (2) haemorrhagic and (3) unknown, according to VARC-2 consensus. ${ }^{39}$ The diagnosis and nature of stroke will be supported by CT or MRI imaging and confirmed by a consultant neurologist.

Table 3 Definition criteria of type $\mathrm{V}$ myocardial infarction

\section{Baseline condition Definition}

1. In patients with normal baseline CK$\mathrm{MB}$ or $\mathrm{CTn}$ (I or T)

The peak CK-MB measured within 48 hours of the procedure rises to $\geq 10 \times$ the local laboratory upper limit of normal (ULN) or to $\geq 5 \times$ ULN with new pathological Q-waves in $\geq 2$ contiguous leads or new persistent LBBB, OR in the absence of CK$\mathrm{MB}$ measurements and a normal baseline cTn, a cTn (I or T) level measured within 48 hours of the procedure rises to $\geq 70 \times$ the local laboratory ULN, or $\geq 35 \times U L N$ with new pathological $\mathrm{Q}$-waves in $\geq 2$ contiguous leads or new persistent LBBB.

2. In patients with elevated baseline CK- The CK-MB (or cTn) rises by an absolute increment equal to those levels MB (or cTn) in whom the biomarker levels recommended above from the most recent preprocedure level. are stable or falling

3. In patients with elevated CK-MB (or cTn) in whom the biomarker levels have not been shown to be stable or falling
The CK-MB (or cTn) rises by an absolute increment equal to those levels recommended above plus new ST-segment elevation or depression plus signs consistent with a clinically relevant MI, such as new onset or worsening heart failure or sustained hypotension.

CK-MB, Creatinine Kinase-MB; cTnl, cardiac troponin I; cTnT, cardiac troponin T; LBBB, left bundle-branch block; MI, myocardial infarction. 
Prolonged use of inotropes ( $>72$ hours)

This refers to the use of inotropes for $>72$ hours after the index operation. The type, dose and duration of administered inotropes will be also collected.

\section{Cardiogenic shock}

Postoperative critical haemodynamic condition requiring mechanical ventricular-assist devices or high-dose inotropes with evidence of peripheral malperfusion. Coexistence of a cardiac index $<1.8 \mathrm{~L} / \mathrm{min} / \mathrm{m}^{2}$ despite adequate correction of all the coexisting preload, afterload, electrolyte and gas-analyses abnormalities will be pursued with the aid of different haemodynamic monitoring methods, according to local Institutional policies (eg, echocardiography, Swan-Ganz catheter, PICCO, PRAM and Vigileo).

\section{Intra-aortic balloon pump}

Intraoperative or postoperative insertion of an IABP device.

\section{Extracorporeal mechanical oxygenation}

Intraoperative or postoperative insertion of an extracorporeal mechanical pump/oxygenation device.

\section{Bleeding}

Classified as (1) life-threatening or disabling bleeding; (2) major bleeding; and (3) minor bleeding, according to the recent definition criteria reported by the VARC-2 document. $^{39}$

\section{Blood loss 12 hours after surgery}

The amount of postoperative blood losses from mediastinal drainages 12 hours after surgery. Intraoperative blood losses are not taken into account. Nadir haemoglobin and nadir haematocrit will be collected.

Number of transfused red blood cell (RBC) units at hospital discharge: total amount of RBC units intraoperatively and/or postoperatively transfused from the beginning of surgery to the day of discharge.

Number of transfused fresh frozen plasma, pooled human plasma (Octaplas) and/or platelets units at hospital discharge: this refers to the transfusion of these blood products from the beginning of surgery to the day of discharge.

\section{Reintervention for bleeding}

Any reoperation for postoperative bleeding, regardless of concomitant haemodynamic problems.

\section{Reintervention for haemodynamic problems}

Any reoperation for haemodynamic instability. This can also be associated with excessive bleeding: in this case, both categories ('Reintervention for bleeding' and 'Reintervention for haemodynamic problems') will be marked.

\section{Pericardial effusion requiring treatment}

Any pericardial effusion requiring interventional treatment (eg, pericardiocentesis, subxifoid dreinage and resternotomy) due to cardiac tamponade, subtamponade or haemodynamic instability refractory to conservative treatment strategies.

Acute renal failure

Severity of acute renal failure after surgery will be graded in AKIN stages from 1 to 3, according to VARC-2 criteria. ${ }^{39}$

\section{Highest postoperative creatinine level}

The highest level of serum creatinine detected after surgery during the inhospital stay. Creatinine levels will be reported in $\mu \mathrm{mol} / \mathrm{L}$.

\section{Renal replacement therapy}

The need for renal replacement therapy will be dichotomised into 'temporary' or 'permanent' (the latter in the event of death while on renal replacement therapy, or if discharged on renal replacement therapy, or in case of life-long need). Type of renal replacement therapy (eg, dialysis, continuous veno-venous hemofiltration (CVVH) and slow continuous ultrafiltration (SCUF)) will be also collected as a note.

\section{Gastrointestinal complications}

Any gastrointestinal complication requiring endoscopy and/or surgical treatment. Endoscopic diagnostic procedures without any associated interventional procedure (diagnostic only) will not fit this definition.

\section{Postoperative infection}

Classified as: (1) surgical site infection, (2) organ infection (respiratory, urinary and gastrointestinal infection), (3) systemic infection (sepsis) and (4) index valve/device infection. Wound complications are graded according to the Centre for Disease Control and Prevention definitions of surgical site infections. ${ }^{52}$ Any surgical site infection occurring within 3 months after surgery will be considered as a postoperative wound infection.

\section{Early repeated intervention for index intervention failure}

This refers to any surgical or percutaneous procedure on the aortic valve and/or the coronary arteries, performed during the same hospital stay for any prosthesis-related or graft-related complication. These events will be marked as occurring or not, and further detailed in their nature ('valvular early procedure', 'coronary early procedure' or 'coronary+valvular early procedure'). Further details will be collected as explanatory notes.

\section{Length of stay in the intensive care unit}

Number of hours spent in the intensive care unit from surgery. Readmissions to intensive care unit will be considered and included in the number estimation.

Length of inhospital stay

Number of days spent in the hospital (ICU stay will be added) from the day of surgery to hospital discharge to any other hospital ward, rehabilitation unit or home. 
Drug antithrombotic treatment at discharge

Collected dichotomic (yes/no) for each of the following drugs: (1) vitamin $\mathrm{K}$ antagonists, (2) new oral anticoagulants and (3) antiplatelets. Further details on type and dose of each drug will be added as a note.

\section{Type of discharge}

Discharge will be categorised according to the Italian National Institutes of Health classification, as follows: (1) death; (2) discharged home; (3) discharged to rehabilitation clinic; (4) voluntary discharge; (5) transferred to other hospital for acute complications; (6) transferred to other hospital for other reasons; (7) transferred to rehab/ other hospital for chronic complications; (8) ordinary discharge+nurse assistance at home; and (9) dismissal.

\section{NYHA at follow-up}

NYHA class will be assessed at hospital discharge, at 6 months, 1 year, yearly up to the fifth year follow-up, and then yearly up to follow-up closure (10 years).

\section{Date of events}

During follow-up, the date of each possible event will be collected as ' $\mathrm{dd} / \mathrm{mm} /$ yyyy'.

\section{Follow-up death}

Death occurring after hospital discharge. Further dichotomisation into cardiovascular and all-cause mortality is based on VARC-2 criteria. $^{39}$

\section{Follow-up stroke}

Any focal or global neurological syndrome occurring after discharge and caused by ischaemia and/or haemorrhage not resolving within 24 hours. The diagnosis and nature of stroke will be made on the basis of findings from brain CT, or MRI, or based on the medical report of a consultant neurologist.

\section{Follow-up myocardial infarction}

Any myocardial infarction occurring after discharge and requiring medical, interventional or surgical treatment occurring after discharge.

\section{Follow-up reintervention on the aortic valve}

Reintervention is defined as any surgical or percutaneous interventional treatment that replaces (or repairs) an aortic prosthesis implanted at the time of the index procedure that is dysfunctional for either structural or non-structural reasons.

\section{Follow-up aortic valve-related adverse event}

This includes: (1) embolism; (2) valve thrombosis; (3) bleeding events; (4) structural valve deterioration; (5) paravalvular leakage; (6) operated valve endocarditis; and (7) haemolysis, based on the definitions of current guidelines for reporting mortality and morbidity after cardiac valve interventions. ${ }^{40}$

\section{Follow-up repeated revascularisation}

Any CABG and/or PCI performed after discharge for coronary graft dysfunction and/or valve-related coronary complication. Any revascularisation due to the progression of an untreated subcritical (at the time of the index procedure) coronary target will not be considered.

Need for implantable cardioverter-defibrillator

Collected as a dichotomous variable (yes/no).

\section{Composite outcome}

According to VARC-2 definitions, ${ }^{39}$ this includes: (1) device success (absence of procedural mortality with correct positioning of a single prosthesis and with intended performance of the prosthesis); (2) early safety at 30 days (composite endpoint of all-cause mortality, all strokes, life-threatening bleeding, acute kidney injury stage 2 or 3 , coronary obstruction requiring intervention, major vascular complication or valve-related dysfunction requiring repeat procedure); (3) clinical efficacy after 30 days (composite endpoint of all-cause mortality, all strokes, hospitalisation for valve-related symptoms or worsening congestive heart failure, NYHA class III or IV and valve-related dysfunction); (4) time-related valve safety (composite endpoint of structural valve deterioration requiring repeat procedure, prosthetic valve endocarditis, thrombosis, thromboembolic events or valve-related VARC bleeding).

\section{Follow-up MACCE}

Defined as a composite endpoint occurring after the 30-day time-point (considered as hospitalisation, 30th day if discharged home or during 'rehab-hospitalisation' at any time-point if never discharged home) and including any of the following adverse events: death from cardiovascular cause, stroke, myocardial infarction and follow-up repeated revascularisation.

\section{Assessment of post procedural aortic prostheses performance}

Data on valve and prosthetic performances will be recorded according to medical reports from a consultant echocardiographer. Data will be collected before surgery, before hospital discharge, at 30 days after surgery, 6 months, 1 year after implantation, yearly thereafter up to the follow-up closure (10th year). Data collected at echocardiographic examination are based on VARC-2 criteria $^{39}$ and aimed at exploring prosthetic valve performance and ventricular performance. A minimum set of echocardiographic data will be considered, as follows: (1) LV function (EF\% based on Simpson's method); (2) indexed LV end-diastolic and end-systolic volumes and diameters; (3) wall motion score index; (4) indexed left atrial volume; (5) indexed LV mass; (6) native valve and prosthetic valve stenotic indexes (peak velocity, mean gradient, Doppler velocity index, effective orifice area and indexed effective orifice area); and (7) native valve and prosthetic valve regurgitation grade (defined as mild, moderate or severe based on several different echocardiographic indexes as regurgitant volume, 


\section{Box 1 Short-Form-8 Health Survey}

Date Name

This survey asks for your views about your health. This information will help you keep track of how you feel and how well you are able to do your usual activities. Answer every question by selecting the answer as indicated. If you are unsure about how to answer a question, please give the best answer you can. For each of the following questions, please mark an $[\mathrm{x}]$ in the one box that best describes your answer. Overall, how would you rate your health during the past 4 weeks? Excellent Very Good Good Fair Poor Very Poor

During the past 4 weeks, how much did physical health problems limit your physical activities (such as walking or climbing stairs)? Not at all Very little Somewhat Quite a lot Could not do physical activities

- During the past 4 weeks, how much difficulty did you have doing your daily work, both at home and away from home, because of your physical health?

Not at all Very little Somewhat Quite a lot Could not do daily work

- How much bodily pain have you had during the past 4 weeks?

None Very mild Mild Moderate Severe Very severe

D During the past 4 weeks, how much energy did you have?

Very much Quite a lot Some A little None

- During the past 4 weeks, how much did your physical health or emotional problems limit your usual social activities with family or friends?

Not at all Very little Somewhat Quite a lot Could not do social activities

- During the past 4 weeks, how much have you been bothered by emotional problems (such as feeling anxious, depressed or irritable)?

Not at all Slightly Moderately Quite a lot Extremely

- During the past 4 weeks, how much did personal or emotional problems keep you from doing your usual work, school or other daily activities?

Not at all Very little Somewhat Quite a lot Could not do daily activities

regurgitant fraction, effective regurgitant orifice area and so on based on local institutional policies). Further assessment of 'intra-prosthetic', 'peri-prosthetic' or 'combined intra+peri-prosthetic' regurgitation will be added.

\section{SF-8 Health Survey questionnaire}

This will be based on eight questionnaire items reported in box $1{ }^{53}$ This examination will be administered before surgery, at hospital discharge, at 30 days, at 6 months, at 1 year, yearly thereafter up to the fifth year of follow-up, then at follow-up closure (10th year).

\section{Ethics and dissemination}

The study is approved by the local institutional review boards/ethical committees according to local or national guidelines for approval of registry studies. Patient's informed consent will be always obtained.

This multicentre, prospective open registry is designed with the aim of investigating a number of controversial issues regarding current treatment options and risk factors for the surgical therapy of SAVS with or without CAD. Several studies and information are expected to derive from the data collected in the registry. These data will provide further knowledge on the mechanisms leading to adverse events during or after surgery for SAVS and help their prevention, thus allowing a 'tailored' surgical approach for the treatment of this disease.

Several studies are planned at the moment:

Primary study:

1. A 5-year study comparing all-cause mortality between SAVR and surgical TAVR. We expect to report a $10 \%$ superiority of SAVR versus TAVR according to sample size calculation and literature data. ${ }^{736}$ Either adjusted and unadjusted analyses will be performed, although the adjusted analysis will be of clearer value, given the expected different risk profiles of SAVR and TAVR patients. Adjustment will be made by entering as covariates all demographic and anthropometric data, risk scores, comorbidities and those baseline characteristics (key echocardiographic and surgical/technical factors included) having a different distribution at univariate analyses between the two patient populations. This study will also report echocardiographic data, functional status, QoL, incidence of cardiovascular mortality, reinterventions on the aortic valve and incidence of structural valve deterioration between 'all-comers' surgical TAVR and SAVR. The study is expected 6 years after the start of data collection, and it is aimed at being presented in a major European cardiology journal.

Secondary substudies

2. An observational study providing results of the different surgical techniques to treat SAVS-in terms of 'all-cause' and 'cardiovascular' mortality, major morbidity and VARC-2 follow-up outcome analysis-at the end of the fifth year follow-up of the last patient enrolled. We aim at present this study in a major cardiothoracic surgical congress and publish it in a congress satellite journal. This study is expected after 6 years from the start of data collection.

3. A study comparing early and 5-year follow-up outcome of mechanical versus biological prostheses in young population ( $<70$ years of age). Propensity-matching and risk-adjusted analyses will be performed. It is aimed at being presented in a major American journal of the cardiology field. This study is expected after 6 years from the start of data collection.

4. A study comparing early and 5-year follow-up outcome of stented versus stentless versus sutureless bioprostheses versus surgical TAVR in small annuli $(\leq 21 \mathrm{~mm})$. Propensity-matching and risk-adjusted analyses will be performed. Post hoc analysis will help elucidate between-group differences. It is aimed at being presented in a major European cardiology journal of the cardiology field. This study is expected after 6 years from the start of data collection.

5. A study comparing early and 5-year follow-up outcome of sutured (both stented and stentless) bioprostheses versus sutureless bioprostheses. Propensity-matching and risk-adjusted analyses will be performed. It is aimed at being presented in a major American or European journal of the cardiology field. This 
study is expected after 6 years from the start of data collection.

6. A 5-year outcome study comparing SAVR versus surgical TAVR in intermediate-risk patients. Propensity-matching and risk-adjusted analyses will be performed. This study is aimed at being presented in a major European journal of the cardiology field. It is expected after 6 years from the start of data collection.

7. A 3-year outcome study comparing different surgical techniques of TAVR (ie, transapical vs transaortic vs transaxillary approach). Propensity-matching and risk-adjusted analyses will be executed if baseline differences are identified between the three subpopulations. Post hoc statistical analyses will identify outcome differences between the three subgroups. This study is aimed at being presented in a European journal of the field. This study is expected after 4 years from the start of data collection.

8. A 5-year outcome study resembling the previous one for final outcome data. This study is aimed at being presented in a major American journal of the field. It is expected after 6 years from the start of data collection.

9. An interim study analysing 30-day outcome of the first 4000 patients enrolled. This study is expected after 1 year from the start of data collection. It is aimed at being presented in a major European cardiothoracic meeting and satellite journal.

10. A study analysing 30-day outcome and 1-year follow-up outcome of the first 4000 SAVR patients enrolled. Subgroup analyses will be aimed at compare different surgical accesses (ie, sternotomy vs ministernotomy vs minithoracotomy). Propensity-matching, risk-adjusted and post hoc analyses will be done appropriately to nullify potential bias in the interpretation of the results and to compare the results of each surgical subgroup. This study is expected after 2 years from the start of data collection. It is aimed at being presented in a major European cardiothoracic congress and satellite journal.

11. A study analysing the 5-year outcome after SAVR + CABGversus TAVR \pm PCI (regardless of the surgical access for SAVR and TAVR) in patients admitted with contemporary critical aortic stenosis and coronary disease. Propensity-score and risk-adjusted analyses will be done as appropriate for a correct interpretation of data. Particular attention will be focused on the role of 'incomplete revascularisation' and of different techniques of 'staged TAVR and PCI' in the transcatheter group. This study is expected after 6 years from the start of data collection. It is aimed at being presented in a major European cardiothoracic congress and satellite journal.

12. A 10-year study comparing all-cause and cardiovascular mortality, echocardiographic data, functional status, QoL, incidence of reinterventions on the aortic valve and incidence of structural valve deterioration between SAVR and surgical TAVR. This study is expected after 11 years from the start of data collection, and it is aimed at being presented in a major European cardiology journal.

13. A 10-year study comparing all-cause and cardiovascular mortality, echocardiographic data, functional status, QoL, incidence of reinterventions on the aortic valve and incidence of structural valve deterioration between SAVR with 'sutured' valves and SAVR with 'sutureless' valves. This study is expected after 11 years from the start of data collection, and it is aimed at being presented in a major cardiology journal.

For all the above-mentioned studies considering propensity score method, demographic data, gender, EuroSCORE-2, STS score, NYHA functional class, frailty scale, LV function, comorbidities and all those baseline characteristics (anthropometric data, key laboratory tests, echocardiographic parameters and surgical factors included) having a different distribution at univariate analyses between the two patient populations and/or potentially acting as bias will be included in the derivation of the propensity score. Furthermore, the variable "participating centre' will always enter the propensity score model, in order to account for 'undetectable' differences between participating centres (eg, perioperative care protocols, institutional protocols, ethnicity and environmental factors not collected in the Registry). Subsequent analyses with matched cohorts will be performed accounting for the matched nature of the data.

Further studies aimed at peculiar subgroup analyses are not considered at this moment, but the E-AVR Steering Committee will evaluate any study/substudy proposal from any researcher involved in the Registry, and accept/ reject it by vote after review and discussion about its feasibility. Therefore, research findings from the E-AVR registry will be disseminated among the scientific community. They will be presented at international congresses and published in peer-reviewed international journals in the fields of cardiac surgery and cardiology. Any formal presentation or publication of data will be considered as a joint publication by the participating physician(s) and will follow the recommendations of the International Committee of Medical Journal Editors (ICMJE) for authorship. Data collection, analysis and writing process will be monitored by the Steering Committee of the E-AVR, which is made up of the PI and a local representing member from each of the participating centres. It is expected that periodical E-AVR Steering Committee meetings will occur, every 6 months for the first 2 years, yearly thereafter up to the end of follow-up. A complete list of the E-AVR Collaborators is reported in online supplementary appendix. The members of the Steering Committee will take responsibility for the quality of data through local audit.

Investigators will be eligible for authorship if they contribute substantially to study planning, data collection, data analysis and interpretation and writing and critical review of the manuscripts. Two authors per centre will 
be included as main authors of each study. As a member of the Steering Committee, the local representing member will take any decisions on coauthorship related to his/her centre on the basis of the above criteria. Those researchers who plan a substudy, interpret the analysis and write the article will be the first and last authors of the study. Analyses will be performed and/or monitored by an independent Central Core Lab. When an article is submitted to a journal with a maximum number of coauthors, the Steering Committee will decide on the authors on the basis of their contribution to the design of the study, data collection, interpretation of data, writing and critical review of the paper.

In the event of future merging with other contemporary registries (eg, collecting data on concurrent interventional-ie, percutaneous transfemoral, transcarotid or trans-axillary-TAVR procedures), the coauthorship of comparative studies (eg, between surgical and interventional treatments) will be defined by the Steering Committees of the different registries involved. However, data will not be made available for sharing until after publication of the principal results of the study. Thereafter, anonymised individual patient data will be made available for secondary research, conditional on assurance from the secondary researcher that the proposed used of the data is compliant with the MRC Policy on Data Preservation and Sharing regarding scientific quality, ethical requirements and value for money. Anonymised data will be shared as long as the patient has agreed and consented to this. A minimum requirement with respect to scientific quality will be a publicly available prespecified protocol describing the purpose, methods and analysis of the secondary research.

\section{Author affiliations}

${ }^{1}$ Division of Cardiac Surgery, University of Verona Medical School, Verona, Italy ${ }^{2}$ Division of Cardiac Surgery, Department of Cardiosciences, Hospital S. CamilloForlanini, Rome, Italy

${ }^{3}$ Department of Cardiovascular Surgery and Anesthesia and Critical Care of Glenfield Hospital, University Hospitals of Leicester NHS Trust, Leicester, UK ${ }^{4}$ Department of Cardiovascular Surgery, University Heart Center Hamburg, Hamburg, Germany

${ }^{5}$ Cardiovascular Surgery, Univeristy Hospital Clinic, Barcelona, Cataluna, Spain

${ }^{6}$ Cardiac Surgery Unit, University of Genova, Genoa, Italy

${ }^{7}$ Division of Cardiac Surgery, Department of Cardiothoracic Sciences, Second University of Naples, Naples, Italy

${ }^{8}$ Cardiothoracic Department, University Hospital of Udine, Udine, Italy

${ }^{9}$ Cardiac Surgery Unit, Centre Hopitalier Universitaire Vaudois, Lausanne,

Switzerland

${ }^{10}$ Division of Cardiac Surgery, St. Barth Hospital NHS, London, UK

${ }^{11}$ Department of Thoracic and Cardio-Vascular Surgery, University Hospital Jean

Minjoz, Besançon, France

${ }^{12}$ Division of Cardiac Surgery, Ospedali Riuniti, Trieste, Italy

${ }^{13}$ Chirurgie Thoracique et Cardio-Vasculaire, Pole TCVN, Hopital Robert Debrè,

Reims, France

${ }^{14}$ Cardiovascular Center, Klinikum Nürnberg, Paracelsus Medical University,

Nuremberg, Germany

${ }^{15}$ Department of Cardiac Surgery, Torino University Hospitals, Turin, Italy

${ }^{16}$ Unit for Clinical Research and Biostatistics, Verona University Hospital, Verona, Italy

${ }^{17}$ Divison of Cardiac Surgery, University of Parma, Parma, Italy
Correction notice This article has been corrected since it first published. The middle name 'Giovanni' has been added in for author 'Vito Giovanni Ruggieri'.

Acknowledgements We would like to thank Lois Clegg, English language teacher at the University of Parma, for her assistance in the revision of the manuscript. We would like to thank Giulia Bisoffi, chief of the Unit for Clinical Research and Biostatistics, Verona University Hospital in Borgo Trento, Verona, Italy, for her statistical assistance and support.

Collaborators Tiziano Gherli, Giuseppe Faggian, Livio San Biagio, Francesco Musumeci, Hermann Reichenspurner, Manuel Castellà, Antonio Salsano, Alessandro Della Corte, Ciro Bancone, Ugolino Livi, Nicola Masala, Gavin J Murphy, Sidney Chocron, Giuseppe Gatti, Luca Maschietto; Stefano Salizzoni, Francesco Pollari, Alessandro Di Cesare, Giulia Bisoffi.

Contributors F0: conception of the study, design of the study, database building, drafting the paper, critically revising the paper, final approval of the version to be published and agreement to be accountable for all aspect of the study. RG, FS, MDF, SS and AndP: conception and design of the study, drafting the paper, critically revising the paper, final approval of the version to be published and agreement to be accountable for all aspect of the study. GM: design of the study, database building, drafting the paper, critically revising the paper, final approval of the version to be published and agreement to be accountable for all aspect of the study. EG and EQ: design of the study, drafting the paper, critically revising the paper, final approval of the version to be published and agreement to be accountable for all aspect of the study. PT, MB, AniP and MR: conception of the study, drafting the paper, critically revising the paper, final approval of the version to be published and agreement to be accountable for all aspect of the study. VGR: conception of the study, critically revising the paper, final approval of the version to be published and agreement to be accountable for all aspect of the study. GS: drafting the paper, critically revising the paper, final approval of the version to be published and agreement to be accountable for all aspects of the study. SR: design of the study, database building, drafting the paper, critically revising the paper, final approval of the version to be published, agreement to be accountable for all aspect of the study and ClinicalTrials.gov publishing. FN: conception of the study, design of the study, database building, drafting the paper, critically revising the paper, final approval of the version to be published, agreement to be accountable for all aspect of the study and ClinicalTrials.gov publishing.

Funding This research received no specific grant from any funding agency in the public, commercial, or not- for-profit sectors.

Competing interests None declared.

Patient consent Obtained.

Ethics approval Ethical Committee University of Parma.

Provenance and peer review Not commissioned; externally peer reviewed.

Open Access This is an Open Access article distributed in accordance with the Creative Commons Attribution Non Commercial (CC BY-NC 4.0) license, which permits others to distribute, remix, adapt, build upon this work non-commercially, and license their derivative works on different terms, provided the original work is properly cited and the use is non-commercial. See: http://creativecommons.org/ licenses/by-nc/4.0/

(C) Article author(s) (or their employer(s) unless otherwise stated in the text of the article) 2018. All rights reserved. No commercial use is permitted unless otherwise expressly granted.

\section{REFERENCES}

1. lung B, Baron G, Butchart EG, et al. A prospective survey of patients with valvular heart disease in Europe: the euro heart survey on valvular heart disease. Eur Heart J 2003;24:1231-43.

2. Vahanian A, Baumgartner $\mathrm{H}$, Bax J, et al. Guidelines on the management of valvular heart disease: the task force on the management of valvular heart disease of the European Society of Cardiology. Eur Heart J 2007;28:230-68.

3. Filsoufi F, Rahmanian PB, Castillo JG, et al. Excellent early and late outcomes of aortic valve replacement in people aged 80 and older. $J$ Am Geriatr Soc 2008;56:255-61.

4 Grossi EA, Schwartz CF, Yu PJ, et al. High-risk aortic valve replacement: are the outcomes as bad as predicted? Ann Thorac Surg 2008;85:102-7.

5. Smith CR, Leon MB, Mack MJ, et al. Transcatheter versus surgical aortic-valve replacement in high-risk patients. $N$ Engl $\mathrm{J} \mathrm{Med}$ 2011;364:2187-98. 
6. Leon MB, Smith CR, Mack M, et al. Transcatheter aortic-valve implantation for aortic stenosis in patients who cannot undergo surgery. N Engl J Med 2010;363:1597-607.

7. Mack MJ, Leon MB, Smith CR, et al. 5-year outcomes of transcatheter aortic valve replacement or surgical aortic valve replacement for high surgical risk patients with aortic stenosis (PARTNER 1): a randomised controlled trial. Lancet 2015;385:2477-84

8. Van Belle E, Juthier F, Susen S, et al. Postprocedural aortic regurgitation in balloon-expandable and self-expandable transcatheter aortic valve replacement procedures: analysis of predictors and impact on long-term mortality: insights from the FRANCE2 Registry. Circulation 2014;129:1415-27.

9. Reardon MJ, Adams DH, Kleiman NS, et al. 2-year outcomes in patients undergoing surgical or self-expanding transcatheter aortic valve replacement. J Am Coll Cardiol 2015;66:113-21.

10. Leon MB, Smith CR, Mack MJ, et al. Transcatheter or surgical aortic-valve replacement in intermediate-risk patients. N Engl J Med 2016;374:1609-20.

11. Tamburino C, Barbanti M, D'Errigo P, et al. 1-year outcomes after transfemoral transcatheter or surgical aortic valve replacement: results from the Italian OBSERVANT study. J Am Coll Cardiol 2015;66:804-12.

12. Rosato S, Santini F, Barbanti M, et al. Transcatheter aortic valve implantation compared with surgical aortic valve replacement in lowrisk patients. Circ Cardiovasc Interv 2016;9:e003326.

13. Shrestha M, Folliguet T, Meuris B, et al. Sutureless perceval $S$ aortic valve replacement: a multicenter, prospective pilot trial. J Heart Valve Dis 2009;18:698-702.

14. Barnhart GR, Accola KD, Grossi EA, et al. TRANSFORM (Multicenter experience with rapid deployment edwards INTUITY valve system for aortic valve replacement) US clinical trial: performance of a rapid deployment aortic valve. J Thorac Cardiovasc Surg 2017;153:241-51.

15. Martens S, Sadowski J, Eckstein FS, et al. Clinical experience with the ATS $3 f$ Enable $₫$ Sutureless Bioprosthesis. Eur J Cardiothorac Surg 2011;40:749-55.

16. Kocher AA, Laufer G, Haverich A, et al. One-year outcomes of the Surgical Treatment of Aortic Stenosis With a Next Generation Surgical Aortic Valve (TRITON) trial: a prospective multicenter study of rapid-deployment aortic valve replacement with the EDWARDS INTUITY valve system. J Thorac Cardiovasc Surg 2013;145:110-6.

17. Dalén M, Biancari F, Rubino AS, et al. Aortic valve replacement through full sternotomy with a stented bioprosthesis versus minimally invasive sternotomy with a sutureless bioprosthesis. Eur $J$ Cardiothorac Surg 2016;49:220-7.

18. Dalén M, Biancari F, Rubino AS, et al. Ministernotomy versus full sternotomy aortic valve replacement with a sutureless bioprosthesis: a multicenter study. Ann Thorac Surg 2015;99:524-30.

19. Biancari F, Barbanti M, Santarpino G, et al. Immediate outcome after sutureless versus transcatheter aortic valve replacement. Heart Vessels 2016;31:427-33.

20. Santarpino G, Pfeiffer S, Jessl J, et al. Sutureless replacement versus transcatheter valve implantation in aortic valve stenosis: a propensity-matched analysis of 2 strategies in high-risk patients. $J$ Thorac Cardiovasc Surg 2014;147:561-7.

21. McClure RS, McGurk S, Cevasco M, et al. Late outcomes comparison of nonelderly patients with stented bioprosthetic and mechanical valves in the aortic position: a propensity-matched analysis. J Thorac Cardiovasc Surg 2014;148:1931-9.

22. Bourguignon T, Bouquiaux-Stablo AL, Candolfi P, et al. Very longterm outcoMes of the carpentier-edwards perimount valve in aortic position. Ann Thorac Surg 2015;99:831-7.

23. Bourguignon $\mathrm{T}$, Lhommet $\mathrm{P}$, El Khoury $\mathrm{R}$, et al. Very long-term outcomes of the carpentier-edwards perimount aortic valve in patients aged 50-65 years. Eur J Cardiothorac Surg 2016;49:1462-8.

24. Vahanian A, Alfieri O, Andreotti $F$, et al. Guidelines on the management of valvular heart disease (version 2012): the joint task force on the management of valvular heart disease of the European Society of Cardiology (ESC) and the European Association for Cardio-Thoracic Surgery (EACTS). Eur J Cardiothorac Surg 2012;42:S1-44.

25. Barbanti M, Webb JG, Tamburino C, et al. Outcomes of redo transcatheter aortic valve replacement for the treatment of postprocedural and late occurrence of paravalvular regurgitation and transcatheter valve failure. Circ Cardiovasc Interv 2016;9:e003930.

26. Del Trigo M, Muñoz-Garcia AJ, Wijeysundera HC, et al. IncidEnce, timing, and predictors of valve hemodynamic deterioration after transcatheter aortic valve replacement: multicenter registry. J Am Coll Cardiol 2016;67:644-55.
27. Onorati F, Biancari F, De Feo M, et al. Mid-term results of aortic valve surgery in redo scenarios in the current practice: results from the multicentre European RECORD (REdo Cardiac Operation Research Database) initiativet. Eur J Cardiothorac Surg 2015;47:269-80.

28. Kaneko T, Loberman D, Gosev I, et al. Reoperative aortic valve replacement in the octogenarians-minimally invasive technique in the era of transcatheter valve replacement. J Thorac Cardiovasc Surg 2014;147:155-62.

29. Tourmousoglou C, Rao V, Lalos S, et al. What is the best approach in a patient with a failed aortic bioprosthetic valve: transcatheter aortic valve replacement or redo aortic valve replacement? Interact Cardiovasc Thorac Surg 2015;20:837-43.

30. O'Sullivan CJ, Stefanini GG, Stortecky S, et al. Coronary revascularization and TAVI: before, during, after or never? Minerva Med 2014;105:475-85.

31. Virk SA, Tian DH, Liou K, et al. Systematic review of percutaneous coronary intervention and transcatheter aortic valve implantation for concomitant aortic stenosis and coronary artery disease. Int $J$ Cardiol 2015;187:453-5

32. Cheung A, Hon JK, Ye J, et al. Combined off-pump transapical transcatheter aortic valve implantation and minimally invasive direct coronary artery bypass. J Card Surg 2010;25:660-2.

33. Curzen N, Diprose P, George W, et al. Novel treatment for critical aortic stenosis with severe aortic root calcification and coronary disease: combined left internal thoracic artery graft to left anterior descending coronary artery and deployment of transcatheter aortic valve implantation valve under direct vision. $J$ Thorac Cardiovasc Surg 2013;146:e53-e54.

34. Goel SS, Ige M, Tuzcu EM, et al. Severe aortic stenosis and coronary artery disease-implications for management in the transcatheter aortic valve replacement era: a comprehensive review. J Am Coll Cardiol 2013;62:1-10.

35. Hickey GL, Grant SW, Cosgriff R, et al. Clinical registries: governance, management, analysis and applications. Eur $J$ Cardiothorac Surg 2013;44:605-14.

36. Groh MA, Diaz I, Johnson AM, et al. Is surgical intervention the optimal therapy for the treatment of aortic valve stenosis for patients with intermediate society of thoracic surgeons risk score? Ann Thorac Surg 2017;103:1193-8.

37. Thourani VH, Suri RM, Gunter RL, Phil D, et al. Contemporary realworld outcomes of surgical aortic valve replacement in 141,905 low-risk, intermediate-risk, and high-risk patients. Ann Thorac Surg 2015;99:55-61.

38. Armitage $\mathrm{P}, \mathrm{McPherson} \mathrm{CK}$, Rowe $\mathrm{BC}$. Repeated significance tests on accumulating data. $J R$ Stat Soc Ser A 1969;132:235-44.

39. Kappetein AP, Head SJ, Généreux P, et al. Updated standardized endpoint definitions for transcatheter aortic valve implantation: the Valve Academic Research Consortium-2 consensus document (VARC-2). Eur J Cardiothorac Surg 2012;42:S45-S60.

40. Akins CW, Miller DC, Turina MI, et al. Guidelines for reporting mortality and morbidity after cardiac valve interventions. $J$ Thorac Cardiovasc Surg 2008;135:732-8.

41. Thygesen K, Alpert JS, Jaffe AS, et al. Third universal definition of myocardial infarction. J Am Coll Cardiol 2012;60:1581-98.

42. Levey AS, Bosch JP, Lewis JB, et al. A more accurate method to estimate glomerular filtration rate from serum creatinine: a new prediction equation. Modification of diet in renal disease study group. Ann Intern Med 1999;130:461-70.

43. Nashef SA, Roques F, Sharples LD, et al. EuroSCORE II. Eur J Cardiothorac Surg 2012;41:734-45.

44. Cockcroft DW, Gault MH. Prediction of creatinine clearance from serum creatinine. Nephron 1976;16:31-41.

45. Child CG, Turcotte JG. Surgery and portal hypertension. In: Child CG, ed. The liver and portal hypertension. Philadelphia: Saunders, 1964:50-64.

46. Habib G, Lancellotti P, Antunes MJ, et al. 2015 ESC Guidelines for the management of infective endocarditis: the task force for the management of infective endocarditis of the European Society of Cardiology (ESC). Endorsed by: European Association for CardioThoracic Surgery (EACTS), the European Association of Nuclear Medicine (EANM). Eur Heart $J$ 2015;36:3075-128.

47. Little Brown. The criteria committee of the New York heart association nomenclature and criteria for diagnosis of diseases of the heart and blood vessels. Boston: Little Brown, 1964.

48. Nishimura RA, Otto CM, Bonow RO, et al. 2014 AHA/ACC guideline for the management of patients with valvular heart disease: executive summary: a report of the American college of cardiology/American heart association task force on practice guidelines. J Am Coll Cardiol 2014:63:2438-88. 
49. Rockwood K, Stadnyk K, MacKnight C, et al. A brief clinical instrument to classify frailty in elderly people. Lancet 1999;353:205-6.

50. Shih T, Paone G, Theurer PF, et al. The society of thoracic surgeons adult cardiac surgery database version 2.73: more is better. Ann Thorac Surg 2015;100:516-21.

51. Moussa ID, Klein LW, Shah B, et al. Consideration of a new definition of clinically relevant myocardial infarction after coronary revascularization: an expert consensus document from the Society for Cardiovascular Angiography and Interventions (SCAI). J Am Coll Cardiol 2013;62:1563-70.

52. Mangram AJ, Horan TC, Pearson ML, et al. Guideline for prevention of surgical site infection, 1999. HoSpital infection control practices advisory committee. Infect Control Hosp Epidemiol 1999;20:250-78.

53. Ware JE, Kosinski M, Dewey JE, et al. How to score and interpret single-item health status measures: a manual for users of the SF-8 health survey. Lincoln, RI: Quality Metric Inc, 2001. 